\title{
Current understanding of ferroptosis in the progression and treatment of pancreatic cancer
}

Shi Dong ${ }^{1}$, Xin Li ${ }^{1}$, Wenkai Jiang ${ }^{1}$, Zhou Chen ${ }^{1}$ and Wence Zhou ${ }^{2^{*}}$ (D)

\begin{abstract}
Pancreatic cancer is a highly malignant tumour of the digestive tract. Despite advances in treatment, its 5-year survival rate remains low, and its prognosis is the worst among all cancers; innovative therapeutic methods are needed. Ferroptosis is a form of regulatory cell death driven by iron accumulation and lipid peroxidation. Recent studies have found that ferroptosis plays an important role in the development and treatment response of tumours, particularly pancreatic cancer. This article reviews the current understanding of the mechanism of ferroptosis and ferroptosisrelated treatment in pancreatic cancer.
\end{abstract}

Keywords: Ferroptosis, Iron accumulation, Lipid peroxidation, Pancreatic cancer

\section{Introduction}

Pancreatic cancer is a malignant tumour with an extremely high mortality rate. Because of its characteristic late diagnosis, high invasiveness, and distant metastasis, the 5-year survival rate of patients is less than $10 \%$ $[1,2]$. Pancreatic ductal adenocarcinoma (PDAC) will become the second leading cause of cancer death in the United States by 2025 [3]. Ferroptosis is a form of irondependent regulatory cell death caused by excessive lipid peroxidation and subsequent plasma membrane rupture [4-6]. Studies have found that tumour cells require more iron than normal cells and are more susceptible to ferroptosis, making ferroptosis a new target to treat drugresistant cancer [7-9]. Strategies regulating ferroptosis are a current research hot spot that influences the progression and treatment of pancreatic cancer. This article reviews the current understanding of the mechanism of

\footnotetext{
${ }^{*}$ Correspondence: zhouwc@lzu.edu.cn

${ }^{2}$ Department of General Surgery, Gansu Province, The First Hospital of Lanzhou University, No. 1, Donggang West Road, Chengguan District, Lanzhou City 730000, China

Full list of author information is available at the end of the article
}

ferroptosis and ferroptosis-related treatment in pancreatic cancer.

\section{Ferroptosis and its relationship with tumours}

Ferroptosis is a non-apoptotic form of cell death. The concept of ferroptosis was first proposed by Dixon et al. in 2012 [10]. In contrast to apoptosis, autophagy and various forms of necrosis [10-12], ferroptosis is the result of iron accumulation, lipid reactive oxygen species (ROS) generation, and decreased glutathione peroxidase 4 (GPX4) activity [12]. The core of ferroptosis is cell damage caused by iron accumulation and lipid peroxidation [13, 14]. Regarding morphology, ferroptotic cells have characteristic mitochondrial atrophy, increased mitochondrial membrane density and disrupted mitochondrial membrane integrity $[10,14]$. Ferroptosis is regulated by inducers and inhibitors. The inducers mainly include erastin [10], P53 [15], Ras-selective lethal 3 (RSL3) [16] and activating transcription factor 4 (ATF4) [17]. Inhibitors include buthionine sulfoximine (BSO) [18], nuclear factor, erythroid 2-like 2 (NFE2L2) [19], ferrostatin-1 (Fer-1) [10] and microRNA-137(miR-137) [20]. The mechanism of ferroptosis regulation is shown in Fig. 1. 


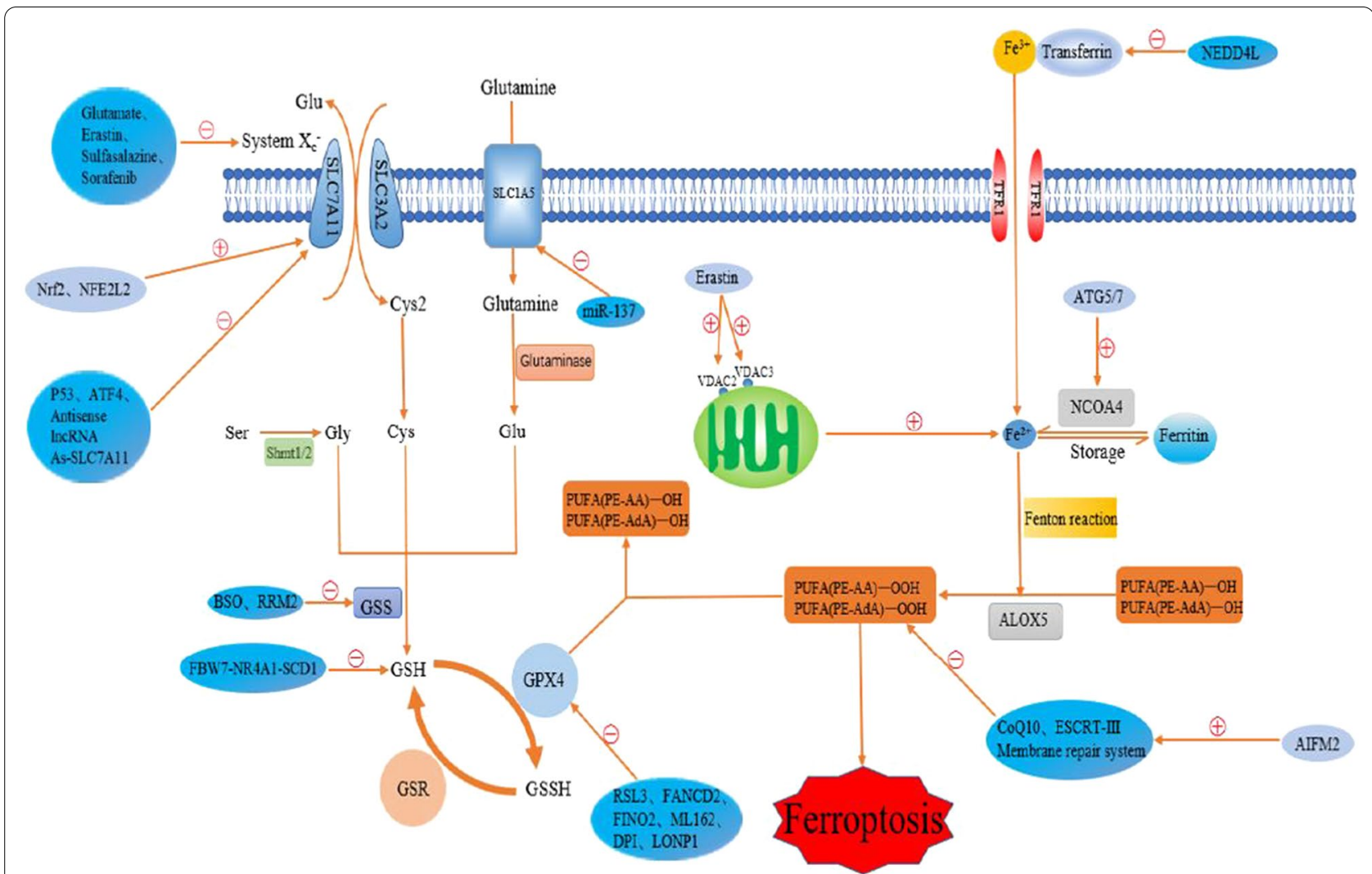

Fig. 1 Mechanisms regulating ferroptosis. Specific mechanisms regulating ferroptosis, including various inducers and inhibitors. System $X_{c}^{-}$ transports extracellular Cys2 into the cell and transports intracellular Glu outside the cell, and then Cys 2 is used to synthesize GSH. GPX4 combines with PUFA-OH to reduce reactive oxygen species generation and ultimately inhibit lipid peroxidation. Extracellular Fe ${ }^{3+}$ binds to the iron transporter and enters the cell through TFR1 on the cell membrane. Next, $\mathrm{Fe}^{3+}$ is reduced to $\mathrm{Fe}^{2+}$ and combined with reactive oxygen species to participate in lipid peroxidation, and finally induce ferroptosis. $\Theta$ indicates inhibition, and $\oplus$ indicates induction, System $X_{c}{ }^{-}$a glutamate/cystine antiporter; Shmt 1/2, serine hydroxymethyltransferase 1/2, GSS glutathione synthase, GSH glutathione, GSSH glutathione persulfide, GPX4 glutathione peroxidase 4, GSR glutathione reductase, ALOX5 arachidonate lipoxygenase 5, NCOA4 nuclear receptor co-activator 4, SLC7A11 solute carrier family 7 member 11, SLC3A2 solute carrier family 3 member 2, SLC1A5 solute carrier family 1 member 5, TFR1 transferrin receptor 1, VDAC2/3 voltage-dependent anion channel 2/3, ATG5/7 autophagy-related protein 5/7, Nrf2 nuclear factor E2-related factor 2, NFE2L2 nuclear factor, erythroid 2-like 2, ATF4 activating transcription factor 4, InCRNA long noncoding RNAs, miRNA microRNA, PUFA polyunsaturated fatty acid, BSO buthionine sulfoximine, RRM2 ribonucleotide reductase M2, FBW7 F-box and WD repeat domain-containing 7, NR4A1 nuclear receptor subfamily 4 group A member 1 , SCD1 stearoyl-CoA desaturase 1, RSL3 Ras-selective lethal 3, FANCD2 Fanconi anaemia complementation group D2, FINO2 1,2-dioxolane, ML162 (S)-enantiomer, DPI diphenylene iodonium, LONP1 mitochondrial Lon protease 1, CoQ10 coenzyme Q10, ESCRT endosomal sorting complex required for transport, AIFM2 apoptosis-inducing factor mitochondria-associated 2

Ferroptosis is widely involved in various physiological activities of the human body, and plays an important role in the occurrence and development of diseases such as cancer, neurodegenerative diseases, and renal failure $[9$, $21,22]$. A summary of studies on the difference between ferroptosis and other types of cell death is shown in Table 1 [23-26].

Main features of ferroptosis, apoptosis, autophagy, necroptosis, necrosis and pyroptosis, including the characteristics, morphological features, biochemical features, regulatory pathways, inducers and inhibitors.

Studies have found that tumour cells are more sensitive to intracellular iron than normal cells [27, 28]. Ferroptosis has been identified in several types of cancers, including gastric cancer [29], lung cancer [30], pancreatic cancer [31], breast cancer [32], melanoma [33] and brain cancer [34]. Studies have confirmed that activating ferroptosis effectively prevents tumour progression and enhances the effects of chemotherapy, radiotherapy and immunotherapy [35-37]. For example, Zhang et al. promoted the ferroptosis of gastric cancer cells by inhibiting miR-522, upregulating the expression of arachidonate lipoxygenase 15 (ALOX15) and inhibiting gastric cancer. This strategy also improved the therapeutic effect of paclitaxel and cisplatin [38]. Nie et al. found that retinoblastoma ( $\mathrm{Rb})$, nuclear factor erythroid 2-related factor 2 (NRF2) and 


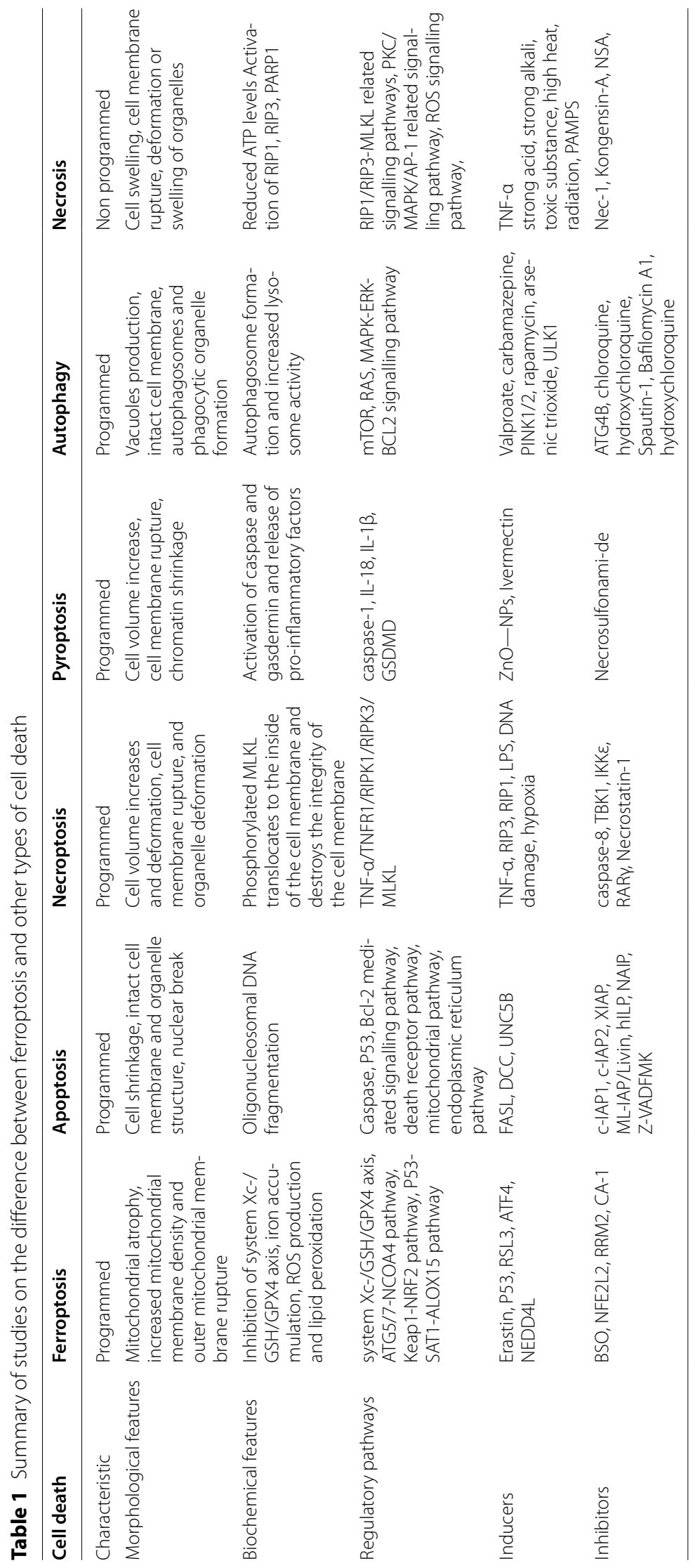


metallothionein-1G (MT-1G) inhibit sorafenib-induced ferroptosis of liver cancer cells and reduce sensitivity to sorafenib [39]. To date, various drugs, such as erastin [10], sorafenib [39], sulfasalazine [40] and glutamate [41], have been developed to induce ferroptosis of tumour cells. The unique molecular mechanism of ferroptosis and its advantages in tumour therapy make it a likely target for cancer treatment.

\section{Mechanism of ferroptosis in the progression of pancreatic cancer}

Since ferroptosis was identified as a novel form of cell death, the role of ferroptosis in cancer has been under extensive study. Studies have found that intervention of the ferroptosis process affects the growth and proliferation of pancreatic cancer cells and progression of pancreatic cancer. [42] This section summarizes the research progress on ferroptosis in pancreatic cancer.

\section{Iron accumulation and pancreatic cancer}

Iron is necessary to maintain cell metabolism. Iron accumulation is an important source of ROS, a key player in ferroptosis, and is closely related to the growth and development of tumours [43, 44]. Wang et al. used erastin and RSL3 to treat pancreatic cancer cell lines (PANC1) and found that NEDD4-like E3 ubiquitin protein ligase (NEDD4L) expression is upregulated. Propidium iodide (PI) staining, revealed that the number of dead cells is increased using specific shRNA to knock out NEDD4L, and the colony formation assay confirmed that NEDD4L knockout enhances tumour suppression induced by erastin and RSL3. The experiment further proved that lactotransferrin (LTF) is the target of NEDD4L, and the decrease in LTF expression significantly inhibites the accumulation of iron in PANC1 cells. Additionally, the production of ROS and malondialdehyde, which is the end product of lipid peroxidation, is reduced, and cell mortality is also reduced. NEDD4L blocks iron accumulation and cell oxidative damage by mediating LTF degradation and ultimately inhibiting tumour growth [45]. As an antimalarial drug, artesunate (ART) inhibits various tumours. Relevant studies have found that pancreatic cancer cell death induced by ART is inhibited by deferoxamine (DFO), while increasing the content of transferrin (HTF) increases the degree of cell death, reflecting that ART-induced cell death depends on the level of free iron. Subsequent experiments proved that ferritin enters the lysosome by binding to NCOA4 and is degraded. At this time, the level of free iron increases, and ART activates lysosome activity by increasing the assembly of V-ATPase and promoting ferritin degradation. Induced ferroptosis plays a role in suppressing cancer [46, 47]. Autophagyrelated genes play a critical role in regulating autophagy and are also closely related to ferroptosis. In one study, inhibiting the expression of the autophagy-related genes autophagy-related protein 5(AGT5) and ATG7 in vitro to prevent the death of erastin-induced pancreatic cancer cell lines (PANC1 and PANC2.03) significantly reduced the ferrous iron and malondialdehyde levels in the cell. The study further proved that nuclear receptor coactivator 4(NCOA4) is a receptor of ATG5 and ATG7 that mediates ferritin degradation and releases ferrous iron. The ATG5-ATG7-NCOA4 autophagy pathway is a new target to treat pancreatic cancer [48].

\section{Oxidative stress and pancreatic cancer}

The activation of oxidative stress and weakening of the antioxidant barrier cause massive ROS production and subsequent lipid peroxidation, which ultimately induces ferroptosis $[49,50]$. In this process, mitochondria, as the power station of cells, play an important role in cell death caused by oxidative stress and ferroptosis. Transcription factor A, mitochondrial (TFAM) is a member of the high mobility group protein family. Loss of its expression causes respiratory chain disorders, mitochondrial dysfunction and oxidative stress. Treatment of PANC1, Capan2 and pHsPDAC cells with the nucleoside analogue Zacitabine led to a decrease in TFAM expression, causing mitochondrial dysfunction in these cells, manifested by a decrease in mitochondrial DNA (mtDNA) replication number and cellular oxygen consumption, limited ATP production and an increase in ROS content, ultimately inducing cell death. Related experiments further proved that Zalcitabine induces ferroptosis by inducing oxidative mtDNA damage and mitochondrial function decline, as well as TFAM degradation and activation of the cyclic GMP-AMP synthase (CGAS) - stimulator of interferon gene 1 (STING1) pathway, thereby inhibiting the growth of pancreatic cancer [51]. Diaphanous homology 3 (DIAPH3) has different functions because of different tumour types. Related databases and clinical tissue sample analysis have found that DIAPH3 is highly expressed in pancreatic cancer and is positively correlated with tumour progression. DIAPH3 also promotes the proliferation and invasion of pancreatic cancer cells. Further mechanistic studies have shown that DIPAH3 inhibition promotes the antioxidant effect mediated by TrxR1 and GPX4, the key factors of selenium metabolism, increases the levels of peroxides and ROS, and ultimately inhibits the malignant phenotype of pancreatic cancer [52]. The magnetic field (MF) is considered to have an anti-tumour effect. Because of its low toxicity and noninvasiveness, MF can be used as an ideal antitumour treatment option. Studies have found that Wilms tumour, lung epithelial cancer, gastric cancer and pancreatic cancer exposed to MF are significantly inhibited 
compared with non-malignant tumours, and the inhibition rate is higher for the third day. Subsequent experiments have shown that MF promotes ROS, increases the NADPH levels, induces cell DNA damage and oxidative stress leading to ferroptosis, and inhibiting tumour proliferation [53]. Although this mechanism has not been verified in pancreatic cancer, it provides a new strategy for subsequent study.

\section{Lipid peroxidation and pancreatic cancer}

Lipid peroxidation is the main characteristic of ferroptosis that is primarily induced by polyunsaturated fatty acids under the action of lipoxygenases and ROS and eventually activates ferroptosis and inhibits tumour development $[54,55]$. Microsomal glutathione S-transferase 1 (MGST1) is a membrane-bound transferase that inhibits oxidative stress and apoptosis. In one study, MGST1 was upregulated in pancreatic cancer cell lines (CFPAC1 and PANC2. 03) treated with erastin and RSL3. Inhibiting the expression of MGST1 increased the level of intracellular malondialdehyde, but did not affect the intracellular iron content, indicating that MGST1 inhibits lipid peroxidation but not iron accumulation. Subsequent studies have found that MGST1 affects lipid peroxidation by regulating ALOX5, and in vivo experiments have confirmed that MGST1 inhibits the growth of xenotransplanted pancreatic cancer mouse tumours. Additionally, by analysing The Cancer Genome Atlas (TCGA) database, the overall survival rate of patients with high MGST1 expression was significantly lower than that of patients with low MGST1 expression, suggesting that MGST1 can be used as an indicator of prognosis for pancreatic cancer patients [56]. As part of the RAS family, ADP ribosylation factor 6 (ARF6) regulates the invasion, metastasis and proliferation of cancer cells, and is closely related to autophagy and immunity. Studies have pointed out that ARF6 is highly expressed in the pancreatic cancer cell lines PANC1 and MIA PaCa-2, and its knockdown promotes RSL3-induced ferroptosis. Further experiments have confirmed that ARF6 does not directly affect lipid peroxidation, but shapes the cell lipid components into a state that is easy to oxidize by regulating the level of ACSL4 protein, and finally induces lipid peroxidation and inhibits pancreatic cancer cell growth [57]. Hu et al. used erastin or RSL3 to treat pancreatic cancer cell lines (PANC1 and MIAPaCa2), which induced lipid peroxidation, pancreatic cancer cell death and low expression of pirin (PIR). Further studies have found that PIR inhibits lipid peroxidation by regulating the expression of acyl-CoA synthetase long-chain family member 4 (ACSL4) and ultimately affecting the tumours growth. PIR prevents the proliferation of pancreatic cancer by negatively regulating ferroptosis [58]. These studies reflect that lipid peroxidation plays an important role in the occurrence and development of pancreatic cancer and providing a new way to treat pancreatic cancer.

\section{System $\mathrm{X}_{\mathbf{c}}{ }^{-}$/GSH/GPX4 axis and pancreatic cancer}

Previous studies have confirmed that cysteine, glutathione (GSH) and GPX4 are the key foctors controlling ferroptosis [59], and system $\mathrm{X}_{\mathrm{c}}^{-}$(a glutamate/cystine antiporter) can ingest cystine, which is ultimately used to synthesize GSH [60]. Ferroptosis is regulated by the system $\mathrm{X}_{\mathrm{c}}{ }^{-} / \mathrm{GSH} / \mathrm{GPX} 4$ axis. Mitochondrial Lon peptidase 1 (LONP1) is a functional enzyme that regulates mitochondrial function and cytological stability. In one study, erastin was used to treat the pancreatic cancer cell line PANC1, and western blot analysis showed that the protein levels of the key ferroptosis factors GPX4 and GSH were significantly decreased, but LONP1 expression was upregulated. Specific shRNAs were then generated to inhibit LONP1 expression. PANC1 cells with LONP1 interference were less sensitive to erastin than control cells, but GPX4 expression and the GSH content were increased; thus, LONP1 was concluded to induce a tumour suppressor effect by downregulating GPX4 expression and reducing the GSH content. Further experiments revealed that LONP1 negatively regulates the nuclear factor E2-related factor 2/Kelch-like ECH-associated protein 1 (Nrf2/Keap1) signalling pathway to affect tumour growth [61]. F-box and WD repeat domain-containing 7 (FBW7) is one of the most commonly mutated genes in human cancers that is expressed at low levels in pancreatic cancer. Ye et al. transfected FBW7 into pancreatic cancer cell lines (PANC1 and SW1990) and detected a decrease in the ratio of GSH to glutathione persulfide (GSSH). They found that intracellular ROS and the malondialdehyde level were increased using the DCFH-DA probe, indicating that FBW7 promotes ROS and lipid peroxidation in pancreatic cancer cells. They further found that FBW7 activates ferroptosis and ultimately inhibits the proliferation of pancreatic cancer cells by regulating nuclear receptor subfamily 4 group A member 1/stearoyl-CoA desaturase 1(NR4A1/SCD1) [62]. In another study, inhibiting GSH synthesis using BSO did not significantly reduce the viability of pancreatic cancer cells. This finding proved that a change in the GSH content alone is insufficient to cause ferroptosis. To further study the mechanism, mass spectrometry was performed, revealing that cystine was not only used to synthesize GSH but also converted into CoA, and the inhibitory system $\mathrm{X}_{\mathrm{c}}^{-}$reduced the level of CoA and increased the content of Coenzyme Q10 (CoQ10), the key substance for CoA synthesis. The tumour cells showed ferroptosis phenotypes such as various lipids, drop formation, matrix rupture and necrosis. Thereafter, the tumour stoppeds 
growing and shrank. Ferroptosis caused by the combined use of pantothenate kinase inhibitor pantothenic acid inhibitors (PANKi) and BSO can be reversed by CoQ10 analogues, confirming that inhibiting GSH and CoA generates the initiation of ferroptosis in pancreatic cancer cells [63]. These studies show that targeting the system $\mathrm{X}_{\mathrm{c}}{ }^{-} / \mathrm{GSH} / \mathrm{GPX} 4$ axis is a potential new research direction for pancreatic cancer treatment.

\section{Ferroptosis and the early diagnosis, prognosis and staging of pancreatic cancer}

Accurately performing the early diagnosis, prognosis and staging in pancreatic cancer will help to better treat and prevent the disease. Pancreatic cancer cells are more dependent on intracellular iron because of their high proliferation and DNA synthesis requirements [45]. The oxidation and reduction of iron promote the generation of oxygen free radicals, which can accelerate tumour growth. Therefore, serum ferritin and transferrin, which reflect the iron level, can be used as potential diagnostic biomarkers for pancreatic cancer detection [64, 65]. Jeong et al. found that knocking out transferrin receptor 1 (TFR1) significantly inhibits the proliferation of PDAC cells, proving that TFR1 is essential for the growth and tumourigenic phenotype of pancreatic cancer, and that it plays a crucial role in the early diagnosis of pancreatic cancer [68]. Ferroptosis can reflect the prognosis and tumour stage of pancreatic cancer patients, and genes related to ferroptosis can be used as biomarkers to predict the average survival time of pancreatic cancer patients. Studies have found that the solute carrier family 7 member 11 (SLC7A11) gene is overexpressed in pancreatic cancer cells, and the survival time of mice with SLC7A11 gene deletion induced by CRISPR-Cas9 is doubled compared with that of normal mice [63]. Other studies have found that the expression of the GPX4 gene in the pancreatic cancer group is upregulated compared with that in the normal group using TCGA database analysis. The overall survival analysis showed that high GPX4 expression is related to the increased survival rate of pancreatic cancer patients (high-expression GPX4 group: $n=131$; low-expression GPX4 group: $n=43$ ). These analyses indicate that GPX4 may be a prognostic marker for pancreatic cancer patients [69]. Additionally, ferroptosis is related to the pancreatic cancer stage. Studies have demonstrated that the expression levels of hepcidin and ferroportin in the tissue samples of pancreatic cancer patients undergoing radical surgery are highly expressed in pancreatic cancer tissues. Low expression of hepcidin and high expression of ferroportin were associated with a poor prognosis of patients, and the hepcidin expression level was associated with the pathological stage and vascular invasion in pancreatic cancer [70].
These results indicate that ferroptosis and related factors are closely related to the early diagnosis, prognosis, and tumour-node-metastasis (TNM) stage of pancreatic cancer, and have attracted widespread attention. Additionally, some researchers have identified 14 potentially valuable genes related to pancreatic cancer prognosis and ferroptosis using Gene Expression Omnibus (GEO) and TCGA databases and have analysed the mRNA and mRNA of these genes in pancreatic cancer tissues using clinical samples. Expression at the protein level confirmed that up-regulated prostaglandin-endoperoxide synthase 2 (PTGS2) expression and down-regulated metallothionein-1G (MT1G), tubulin epsilon 1 (TUBE1) and autophagy-related gene 4D (ATG4D) increase the risk of pancreatic cancer and lead to a poor prognosis; however, further experimental verification is warranted [72]. Ferroptosis is expected to play a crucial role in the comprehensive treatment of pancreatic cancer.

A summary of studies on the molecular markers of ferroptosis related to the diagnosis, prognosis and staging of pancreatic cancer is shown in Table 2.

Summary of the molecular markers of ferroptosis related to pancreatic cancer, including biomolecular types and clinical relevance. The biomolecular types mainly involve DNA, RNA and protein. The clinical relevance mainly includes prognosis, diagnosis, and TNM staging.

\section{Role of ferroptosis in pancreatic cancer treatment} Ferroptosis and targeted therapy of pancreatic cancer

As the role of ferroptosis in pancreatic cancer was gradually uncovered, studies on its applications in the treatment of pancreatic cancer increased. As a stress-induced transcription factor, stress-induced nuclear protein 1 (NUPR1) can prevent ferroptosis. Liu et al. found that the lack of NUPR1 promotes ROS formation induced by erastin and RSL3, and inhibited the viability of pancreatic cancer cells. Further studies have shown that lipocalin 2 (LCN2), as the direct target gene of NUPR1, regulates iron levels in pancreatic cancer cells. By inhibiting the NUPR1-LCN2 pathway, iron accumulation and cell oxidative damage are reduced, promoting the growth of pancreatic cancer. Thus, targeting the NUPR1LCN2 pathway provides a new strategy to treat pancreatic cancer [73]. Kuang et al. found that a lysosomal cysteine protease prevents the translocation of cathep$\sin$ B (CTSB) into the nucleus and causes DNA damage, thereby activating STING1 and ultimately inducing ferroptosis, providing a new way to improve the anticancer effect of ferroptosis [74]. GSH, which plays a key role in ferroptosis, is considered an important target for anticancer therapy, and targeting regulatory factors that affect GSH synthesis also provides a new direction for cancer 
Table 2 Summary of studies on the molecular markers of ferroptosis related to the diagnosis, prognosis and staging of pancreatic cancer

\begin{tabular}{llll}
\hline Molecular marker & Biomolecule type & Clinical relevance & References \\
\hline Ferritin & Protein & Diagnosis, prognosis & {$[65,66]$} \\
Ferroportin & Protein & Diagnosis, prognosis & {$[60,65]$} \\
Hepcidin & Protein & Prognosis, TNM stage & {$[67]$} \\
Lipocalin 2 & Protein & Diagnosis & {$[57,65]$} \\
TFR1 & Protein & Diagnosis, prognosis, TNM stage & {$[63,65]$} \\
SLC7A11 & DNA & Prognosis & {$[70]$} \\
GPX4 & Protein & Prognosis & {$[67]$} \\
ARF6 & Protein & Prognosis & {$[57]$} \\
SLC25A37/28 & DNA & Prognosis & {$[71]$} \\
PARK2 & DNA & Prognosis & {$[71]$} \\
AIM2 & DNA & Prognosis & {$[71]$} \\
NUPR1 & DNA & Prognosis & {$[73]$} \\
LCN2 & DNA & Prognosis & {$[73]$} \\
MGST1 & DNA & Prognosis & {$[56]$} \\
PTGS2 & DNA & Prognosis & {$[72]$} \\
MT1G & DNA & Prognosis & {$[72]$} \\
TUBE1 & DNA & Prognosis & {$[72]$} \\
ATG4D & DNA & Prognosis & {$[72]$} \\
\hline
\end{tabular}

treatment $[75,76]$. Inhibiting SLC7A11 expression are reported to decrease the GSH content, thereby activating ferroptosis, and ultimately inhibiting the proliferation and survival of pancreatic cancer cells [77]. GPX4 is also a key factor in regulating ferroptosis process. Some studies have found that the autophagy inducers rapamycin and RSL3 cause the degradation of human pancreatic cancer cell GPX4 protein. Inhibiting GPX4 expression can enhance the anticancer activity of rapamycin and RSL3 in vivo or in vitro [78]. Presently, studies on the targeted therapy of ferroptosis in pancreatic cancer remain limited. Promoting ferroptosis in a high-glucose environment can kill pancreatic cancer cells more effectively. The pathway involves solute carrier family 2 member 1 (SLC2A1) promoting glucose uptake and inhibiting the expression of pyruvate dehydrogenase kinase 4 (PDK4), which induces lipid peroxidation and ultimately inhibits tumour growth; however, the specific mechanism and role of glucose and lipid metabolism in ferroptosis in pancreatic cancer remain to be further studied [79].

\section{Ferroptosis and immunotherapy of pancreatic cancer}

The tumour microenvironment remains a hot research topic, and immunotherapy has also become a favoured option for cancer treatment. However, pancreatic cancer is not sensitive to immunotherapy [80]. Recent studies have found that immunotherapy is closely related to ferroptosis [38]. Ferroptosis induction to improve the sensitivity of pancreatic cancer to immunotherapy may become a new treatment strategy for pancreatic cancer. High infiltration of tumour-associated macrophages (TAMs) indicates a poor prognosis of pancreatic cancer, and M2-type TAMs can express immunosuppressive signals to promote the proliferation and invasion of pancreatic cancer cells [81]. Dai et al. found that iron and GPX4 regulate macrophage infiltration in the pancreatic tumour microenvironment, and an in vivo experiment showed that a high-iron diet or GPX4 deletion induces transmembrane protein 173(TMEM173) to promote TAM infiltration, leading to pancreatic intraepithelial neoplasia and an increased pancreas weight in mice [69]. Presently, studies on ferroptosis and pancreatic cancer treatment is still limited, and most rely on big data such as GEO and TCGA to speculate the ferroptosis regulatory factors related to pancreatic cancer immune cell infiltration, such as the aforementioned PTGS2 [72], MTIG [72] and keratin 6A (KRT6A) [82], collagen type $\mathrm{V}$ alpha 2 chain (COL5A2) [82], However, further experimental verification is needed, and the mechanism of action of strategies employing ferroptosis and immunotherapy requires further study. Studies on iron metabolism regulation and immune characteristics will provide new research ideas to treat and prevent pancreatic cancer.

\section{Ferroptosis and chemotherapy of pancreatic cancer}

Gemcitabine is a first-line adjuvant chemotherapeutic drug for pancreatic cancer. Studies have indicated that the combined application of chemotherapeutic drugs 
and ferroptosis inducers helps improve the efficiency of chemotherapy [4], providing a new strategy to improve the sensitivity of pancreatic cancer to gemcitabine and enhance the efficacy of gemcitabine. Tang et al. analysed the gene expression profiles of parental pancreatic cancer cell lines and gemcitabine-resistant pancreatic cancer cell lines and found that components of system $\mathrm{X}_{\mathrm{c}}{ }^{-}$, such as SLC7A11 and solute carrier family 3 member 2 (SLC3A2), are significantly upregulated in cells exposed to gemcitabine, indicating that ferroptosis inducers targeting system Xc- may be effective to treat gemcitabineresistant pancreatic cancer [80]. In another study, erastin enhanced the cytotoxic effects of gemcitabine and cisplatin in two pancreatic cancer cell lines by inhibiting SLC7A11 expression and increasing the apoptosis rate, improving the efficacy of chemotherapy drugs [63]. Additionally, ARF6 increases the sensitivity of pancreatic cancer cells to gemcitabine by inhibiting iron metabolism and gemcitabine-related metabolic proteins including deoxycytidine kinase (DCK)/human equilibrative nucleoside transporter 1 (hENT1), but the specific mechanism requires further study [57]. Presently, few studies have investigated the link between ferroptosis and gemcitabine, but their combination is a feasible strategy for the comprehensive treatment of pancreatic cancer.

\section{Ferroptosis and radiotherapy of pancreatic cancer}

In the multimodal treatment of pancreatic cancer, particularly to treat locally advanced or recurrent pancreatic tumours, radiotherapy remains a critical component [83].
Using reasonable intensity rays promotes the ferroptosis of tumour cells and reduce tumour growth, while using ferroptosis inhibitors reduces the curative effect of radiotherapy on tumours, indicating that ferroptosis modifiers can be used as radiosensitizers to improve the efficacy of radiotherapy without increasing the radiation dose and greatly reduce the serious side effects caused by overdose [8, 84]. Some studies have found that erastin, which induces ferroptosis, enhances the sensitivity of breast, cervical and lung cancer cells to radiation and promotes cell death [85-87]. Although current studies on ferroptosis combined with radiotherapy in pancreatic cancer are limited, they suggest that using radiotherapy combined with ferroptosis inducers provides a new method for advanced and recurrent pancreatic cancer.

A summary of studies on the role of ferroptosis in the treatment and drug resistance of pancreatic cancer is shown in Table 3.

\section{Summary and prospects}

Pancreatic cancer remains one of the most difficult malignant tumours. Its characteristics such as high invasiveness and drug resistance have led to its high mortality rate; new treatment methods are needed. With the identification of ferroptosis, its unique molecular mechanism and role in tumours have gradually emerged. The ability of ferroptosis to improve chemotherapy, radiotherapy and immunotherapy is expected to provide new strategies for cancer treatment, particularly in pancreatic cancer [4]. However, studies on ferroptosis in pancreatic

Table 3 Summary of studies on the role of ferroptosis in the treatment and drug resistance of pancreatic cancer

\begin{tabular}{|c|c|c|c|c|c|}
\hline Interventions & Mechanism of action & Application & In vitro/ in vivo & Animal model & References \\
\hline NUPR1-LCN2 & Reduces iron accumulation and inhibits ROS generation & Suppression & Both & athymic nude female mice & {$[73]$} \\
\hline CTSB & Activates STING1 to induce ferroptosis & Suppression & Both & NOD-SCID female mice & {$[74]$} \\
\hline CRISPR-Cas9 & $\begin{array}{l}\text { Inhibits the expression of SLC7A } 11 \text { and reduces the } \\
\text { content of GSH }\end{array}$ & Suppression & Both & female athymic mice & [77] \\
\hline GPX4 & $\begin{array}{l}\text { Rapamycin and RSL } 3 \text { enhance anticancer activity by } \\
\text { inducing GPX4 protein degradation }\end{array}$ & Suppression & Both & - & [78] \\
\hline SLC2A1 & $\begin{array}{l}\text { SLC2A1 promotes glucose uptake and inhibits the } \\
\text { expression of PDK4, which induces lipid peroxidation }\end{array}$ & Suppression & Both & male C57BL/6 J mice & [79] \\
\hline $\begin{array}{l}\text { GPX4 depletion } \\
\text { or high iron diet }\end{array}$ & Activates TMEM173 to promote TAM infiltration & Promotion & Both & C57BL/6 mice & [69] \\
\hline Erastin & $\begin{array}{l}\text { Inhibits SLC7A11 to improve sensitivity to gemcitabine } \\
\text { and cisplatin }\end{array}$ & Suppression & Both & female athymic mice & [63] \\
\hline ARF6 & $\begin{array}{l}\text { Inhibits iron metabolism and improves sensitivity to } \\
\text { gemcitabine }\end{array}$ & Suppression & In vitro & - & [57] \\
\hline NEDD4L & Degrades LTF to inhibit ferroptosis & Promotion & In vitro & - & [45] \\
\hline LONP1 inhibitor & $\begin{array}{l}\text { Activates the Nrf2/Keap } 1 \text { signalling pathway and upregu- } \\
\text { lates GPX4 expression }\end{array}$ & Promotion & In vitro & - & {$[61]$} \\
\hline
\end{tabular}

"Suppression" indicates that the intervention suppresses pancreatic cancer. "Promotion" indicates that the intervention promotes pancreatic cancer. "In vitro/in vivo" indicates whether the study was performed in vivo, in vitro, or both 
cancer remain in their infancy. A comprehensive analysis of ferroptosis-related mechanisms, such as the role of iron accumulation and lipid peroxidation in cell energy metabolism and autophagy, the effect of ferroptosis on pancreatic carcinoma stem cells, and the link between ferroptosis and tumour resistance and immune infiltration is critical. Additionally, future studies should focus more on the applications of ferroptosis in cancer treatment (particularly for pancreatic cancer), such as the following: how ferroptosis-related mechanisms can be targeted to treat pancreatic cancer with glucose and lipid metabolism disorders; and how ferroptosis can be induced or induce the recruitment of macrophages and other immune cells to cancer to play a role in immunotherapy; how iron metabolism affects the sensitivity of gemcitabine in the treatment of pancreatic cancer; the therapeutic effect of ferroptosis regulators combined with magnetic fields or radiation on pancreatic cancer. However, the synergistic effects of ferroptosis-regulating therapy with chemotherapy, immunotherapy and other antitumour strategies are expected to encourage new treatments for pancreatic cancer and other incurable cancers. We believe that ferroptosis and related factors have valuable research prospects in tumour treatment and prognostication.

\footnotetext{
Abbreviations

PDAC: Pancreatic ductal adenocarcinoma; ROS: Reactive oxygen species; GPX4: Glutathione peroxidase 4; RSL3: Ras-selective lethal 3; ATF4: Activating transcription factor 4; BSO: Buthionine sulfoximine; NFE2L2: Nuclear factor, erythroid 2-like 2; Fer-1: Ferrostatin-1; miR-137: MicroRNA-137; ALOX15: Arachidonate lipoxygenase 15; Rb: Retinoblastoma; NRF2: Nuclear factor erythroid 2-related factor 2; MT-1G: Metallothionein-1G; NEDD4L: NEDD4-like E3 ubiquitin protein ligase; LTF: Lactotransferrin; ATG5/7: Autophagy-related protein 5/7; NCOA4: Nuclear receptor coactivator 4; MGST1: Microsomal glutathione S-transferase 1;TCGA: The Cancer Genome Atlas; CGAS: Cyclic GMP-AMP synthase; mtDNA: Mitochondrial DNA; STING1/TMEM173: Stimulator of interferon response CGAMP interactor 1; TFAM: Transcription factor A, Mitochondrial; PIR: Pirin; ACSL4: Acyl-CoA synthetase long-chain family member 4; system $X_{c}{ }^{-}$: The glutamate/cystine antiporter; GSH: Glutathione; LONP1: Mitochondrial Lon peptidase 1; Nrf2: Nuclear factor E2-related factor 2; Keap 1: Kelch-like ECH-associated protein 1; FBW7: F-box and WD repeat domain-containing 7; NR4A1: Nuclear receptor subfamily 4 group A member 1; SCD1: StearoylCoA desaturase 1; GSSH: Glutathione persulfide; TFR1: Transferrin receptor 1; SLC7A11: Solute carrier family 7 member 11; CTSB: Cathepsin B; TAMs: Tumourassociated macrophages; SLC3A2: Solute carrier family 3 member 2; SLC2A1: Solute carrier family 2 member 1; ARF6: ADP-ribosylation factor 6; DCK: Deoxycytidine kinase; hENT1: Human equilibrative nucleoside transporter 1; COQ10: CoenzymeQ10; MAPK: Mitogen-activated protein kinase; MLKL: Mixed lineage kinase domain like protein; mTOR: Mammalian target of rapamycin; PKC: Protein kinase C; RIP: Receptor-interacting serine/threonine kinase; SAT1: Spermidine/spermine N1-acetyltransferase 1; TFR1: Transferrin receptor 1; DIAPH3: Diaphanous homology 3; MF: Magnetic field; PTGS2: Prostaglandinendoperoxide synthase 2; MT1G: Metallothionein-1G; TBUE1: Tubulin epsilon 1; ATG4D: Autophagy-related gene 4D; NUPR1: Nuclear protein 1; LCN2: Lipocalin 2; PDK4: Pyruvate dehydrogenase kinase 4; KRT6A: Keratin 6A; COL5A2: Collagen type $V$ alpha 2 chain.
}

\section{Acknowledgements}

We sincerely appreciate all the participants in our work.

\section{Authors' contributions}

SD: Conceptualization, Writing- Original draft preparation. XL: Writing- Original draft preparation, Visualization. WJ: Writing- Original draft preparation, Visualization. ZC: Writing一Review \& Editing, Supervision. WZ: Writing一Review \& Editing, Supervision, Project administration, Funding acquisition. All authors read and approved the final manuscript."

\section{Funding}

This work was supported by the Science and Technology Projects of Chengguan District in Lanzhou (2020-2-11-4) and Traditional Chinese Medicine Scientific Research Project of Gansu Province (GZKP-2020-28).

Availability of data and materials

All the data are included in the article.

\section{Declarations}

Ethic approval and consent to participate

Not applicable.

\section{Consent for publication}

Not applicable.

\section{Competing interests}

The authors declare that they have no competing interests.

\section{Author details}

${ }^{1}$ The First School of Clinical Medicine, Lanzhou University, Lanzhou, China. ${ }^{2}$ Department of General Surgery, Gansu Province, The First Hospital of Lanzhou University, No. 1, Donggang West Road, Chengguan District, Lanzhou City 730000, China.

Received: 4 June 2021 Accepted: 19 August 2021

Published online: 09 September 2021

\section{References}

1. Golan T, Hammel P, Reni M, et al. Maintenance olaparib for germline BRCA -mutated metastatic pancreatic cancer. N Engl J Med. 2019;381(4):31727. https://doi.org/10.1056/NEJMoa1903387.

2. Versteijne E, Suker M, Groothuis K, et al. Preoperative chemoradiotherapy versus immediate surgery for resectable and borderline resectable pancreatic cancer: results of the dutch randomized phase III PREOPANC trial. J Clin Oncol. 2020;38(16):1763-73. https://doi.org/10.1200/JCO.19.02274.

3. Schnipper J, Dhennin-Duthille I, Ahidouch A, et al. Ion channel signature in healthy pancreas and pancreatic ductal adenocarcinoma. Front Pharmacol. 2020;1 1:568993. https://doi.org/10.3389/fphar.2020.568993 (Published 2020 Oct 16)

4. Chen X, Kang R, Kroemer G, et al. Broadening horizons: the role of ferroptosis in cancer. Nat Rev Clin Oncol. 2021;18(5):280-96. https://doi.org/ 10.1038/s41571-020-00462-0.

5. Dixon SJ, Stockwell BR. The role of iron and reactive oxygen species in cell death. Nat Chem Biol. 2014;10(1):9-17. https://doi.org/10.1038/nchem bio. 1416.

6. Wu J, Minikes AM, Gao M, et al. Intercellular interaction dictates cancer cell ferroptosis via NF2-YAP signalling. Nature. 2019;572(7769):402-6. https://doi.org/10.1038/s41586-019-1426-6 (published correction appears in Nature. 2019 Aug 2;:).

7. Perez MA, Magtanong L, Dixon SJ, Watts JL. Dietary lipids induce ferroptosis in caenorhabditiselegans and human cancer cells. Dev Cell. 2020;54(4):447-454.e4. https://doi.org/10.1016/j.devcel.2020.06.019.

8. Lang X, Green MD, Wang W, et al. Radiotherapy and immunotherapy promote tumoral lipid oxidation and ferroptosis via synergistic repression of SLC7A11. Cancer Discov. 2019;9(12):1673-85. https://doi.org/10.1158/ 2159-8290.CD-19-0338.

9. Lee H, Zandkarimi F, Zhang Y, et al. Energy-stress-mediated AMPK activation inhibits ferroptosis. Nat Cell Biol. 2020;22(2):225-34. https://doi.org/ 10.1038/s41556-020-0461-8. 
10. Dixon SJ, Lemberg KM, Lamprecht MR, et al. Ferroptosis: an iron-dependent form of nonapoptotic cell death. Cell. 2012;149(5):1060-72. https:// doi.org/10.1016/j.cell.2012.03.042.

11. Doll S, Freitas FP, Shah R, et al. FSP1 is a glutathione-independent ferroptosis suppressor. Nature. 2019;575(7784):693-8. https://doi.org/10.1038/ s41586-019-1707-0.

12. Cao JY, Dixon SJ. Mechanisms of ferroptosis. Cell Mol Life Sci. 2016;73(1112):2195-209. https://doi.org/10.1007/s00018-016-2194-1.

13. Hirschhorn T, Stockwell BR. The development of the concept of ferroptosis. Free Radic Biol Med. 2019;133:130-43. https://doi.org/10.1016/j.freer adbiomed.2018.09.043.

14. Zou Y, Henry WS, Ricq EL, et al. Plasticity of ether lipids promotes ferroptosis susceptibility and evasion. Nature. 2020;585(7826):603-8. https://doi. org/10.1038/s41586-020-2732-8.

15. Jiang $\mathrm{L}$, Kon N, LiT, et al. Ferroptosis as a p53-mediated activity during tumour suppression. Nature. 2015;520(7545):57-62. https://doi.org/10. 1038/nature14344.

16. Xu T, Ding W, Ji X, et al. Molecular mechanisms of ferroptosis and its role in cancer therapy. J Cell Mol Med. 2019;23(8):4900-12. https://doi.org/10. 1111/jcmm.14511.

17. Sato $H$, Kuriyama-Matsumura $K$, Hashimoto $T$, et al. Effect of oxygen on induction of the cystine transporter by bacterial lipopolysaccharide in mouse peritoneal macrophages. J Biol Chem. 2001;276(13):10407-12. https://doi.org/10.1074/jbc.M007216200.

18. Sun $Y$, Zheng Y, Wang C, et al. Glutathione depletion induces ferroptosis, autophagy, and premature cell senescence in retinal pigment epithelial cells. Cell Death Dis. 2018;9(7):753. https://doi.org/10.1038/s41419-0180794-4 (Published 2018 Jul 9).

19. Lin $\mathrm{X}$, Yang $\mathrm{H}$, Zhang $\mathrm{H}$, et al. A novel transcription mechanism activated by ethanol: induction of Slc7a11 gene expression via inhibition of the DNA-binding activity of transcriptional repressor octamer-binding transcription factor 1 (OCT-1). J Biol Chem. 2013;288(21):14815-23. https:// doi.org/10.1074/jbc.M113.466565.

20. Luo M, Wu L, Zhang K, et al. miR-137 regulates ferroptosis by targeting glutamine transporter SLC1 A5 in melanoma. Cell Death Differ. 2018;25(8):1457-72. https://doi.org/10.1038/s41418-017-0053-8.

21. Stockwell BR, Friedmann Angeli JP, Bayir $\mathrm{H}$, et al. Ferroptosis: a regulated cell death nexus linking metabolism, redox biology, and disease. Cell. 2017;171(2):273-85. https://doi.org/10.1016/j.cell.2017.09.021.

22. Zhang Y, Shi J, Liu X, et al. BAP1 links metabolic regulation of ferroptosis to tumour suppression. Nat Cell Biol. 2018;20(10):1181-92. https://doi. org/10.1038/s41556-018-0178-0.

23. Oberst A, Dillon CP, Weinlich $R$, et al. Catalytic activity of the caspase8-FLIP(L) complex inhibits RIPK3-dependent necrosis. Nature. 2011;471(7338):363-7. https://doi.org/10.1038/nature09852.

24. Shan B, Pan H, Najafov A, et al. Necroptosis in development and diseases. Genes Dev. 2018;32(5-6):327-40. https://doi.org/10.1101/gad.312561. 118.

25. Yang WS, Stockwell BR. Synthetic lethal screening identifies compounds activating iron-dependent, nonapoptotic cell death in oncogenic-RASharboring cancer cells. Chem Biol. 2008;15(3):234-45. https://doi.org/10. 1016/j.chembiol.2008.02.010.

26. Brown CW, Amante JJ, Chhoy P, et al. Prominin2 drives ferroptosis resistance by stimulating iron export. Dev Cell. 2019;51(5):575-586.e4. https:// doi.org/10.1016/j.devcel.2019.10.007.

27. Camiolo G, Barbato A, Giallongo C, et al. Iron regulates myeloma cell/ macrophage interaction and drives resistance to bortezomib. Redox Biol. 2020;36:101611. https://doi.org/10.1016/j.redox.2020.101611.

28. Campisi A, Bonfanti R, Raciti G, et al. Gene Silencing of transferrin-1 receptor as a potential therapeutic target for human follicular and anaplastic thyroid cancer. Mol Ther Oncolytics. 2020;16:197-206. https://doi.org/10. 1016/j.omto.2020.01.003 (Published 2020 Jan 21).

29. Sun $X$, Yang $S$, Feng $X$, et al. The modification of ferroptosis and abnormal lipometabolism through overexpression and knockdown of potential prognostic biomarker perilipin2 in gastric carcinoma. Gastric Cancer. 2020;23(2):241-59. https://doi.org/10.1007/s10120-019-01004-z.

30. Wang M, Mao C, Ouyang L, et al. Long noncoding RNA LINC00336 inhibits ferroptosis in lung cancer by functioning as a competing endogenous RNA. Cell Death Differ. 2019;26(11):2329-43. https://doi.org/10.1038/ s41418-019-0304-y (published correction appears in Cell Death Differ. 2019 Aug 5;:).
31. Wang K, Zhang Z, Tsai HI, et al. Branched-chain amino acid aminotransferase 2 regulates ferroptotic cell death in cancer cells. Cell Death Differ. 2021;28(4):1222-36. https://doi.org/10.1038/s41418-020-00644-4.

32. Mao C, Wang X, Liu Y, et al. A G3BP1-interacting IncRNA promotes ferroptosis and apoptosis in cancer via nuclear sequestration of p53. Cancer Res. 2018;78(13):3484-96. https://doi.org/10.1158/0008-5472. CAN-17-3454.

33. Gagliardi M, Saverio V, Monzani R, et al. Ferroptosis: a new unexpected chance to treat metastatic melanoma? Cell Cycle. 2020;19(19):2411-25. https://doi.org/10.1080/15384101.2020.1806426.

34. Nagpal A, Redvers RP, Ling X, et al. Neoadjuvant neratinib promotes ferroptosis and inhibits brain metastasis in a novel syngeneic model of spontaneous HER2 ${ }^{\text {+ve }}$ breast cancer metastasis. Breast Cancer Res. 2019;21(1):94. https://doi.org/10.1186/s13058-019-1177-1 (Published 2019 Aug 13).

35. Guo J, Xu B, Han Q, et al. Ferroptosis: a novel anti-tumor action for cisplatin. Cancer Res Treat. 2018;50(2):445-60. https://doi.org/10.4143/crt. 2016.572.

36. Sun X, Niu X, Chen R, et al. Metallothionein-1G facilitates sorafenib resistance through inhibition of ferroptosis. Hepatology. 2016;64(2):488-500. https://doi.org/10.1002/hep.28574.

37. Wang W, Green M, Choi JE, et al. CD8+ T cells regulate tumour ferroptosis during cancer immunotherapy. Nature. 2019;569(7755):270-4. https:// doi.org/10.1038/s41586-019-1170-y.

38. Zhang H, Deng T, Liu R, et al. CAF secreted miR-522 suppresses ferroptosis and promotes acquired chemo-resistance in gastric cancer. Mol Cancer. 2020;19(1):43. https://doi.org/10.1186/s12943-020-01168-8 (Published 2020 Feb 27).

39. Nie J, Lin B, Zhou M, et al. Role of ferroptosis in hepatocellular carcinoma. J Cancer Res Clin Oncol. 2018;144(12):2329-37. https://doi.org/10.1007/ s00432-018-2740-3.

40. Kim EH, Shin D, Lee J, et al. CISD2 inhibition overcomes resistance to sulfasalazine-induced ferroptotic cell death in head and neck cancer. Cancer Lett. 2018;432:180-90. https://doi.org/10.1016/j.canlet.2018.06. 018.

41. Dixon SJ, Patel DN, Welsch M, et al. Pharmacological inhibition of cystineglutamate exchange induces endoplasmic reticulum stress and ferroptosis. Elife. 2014;3:e02523. https://doi.org/10.7554/eLife.02523 (Published 2014 May 20).

42. Du J, Wang X, Li Y, et al. DHA exhibits synergistic therapeutic efficacy with cisplatin to induce ferroptosis in pancreatic ductal adenocarcinoma via modulation of iron metabolism. Cell Death Dis. 2021;12(7):705. https:// doi.org/10.1038/s41419-021-03996-y (Published 2021 Jul 15).

43. Pan $Z Y$, Tan $C P$, Rao $L S$, et al. Recoding the cancer epigenome by intervening in metabolism and iron homeostasis with mitochondria-targeted rhenium(I) complexes. Angew Chem Int Ed Engl. 2020;59(42):18755-62. https://doi.org/10.1002/anie.202008624.

44. Torti SV, Manz DH, Paul BT, et al. Iron and cancer. Annu Rev Nutr. 2018;38:97-125. https://doi.org/10.1146/annurev-nutr-082117-051732.

45. Wang Y, Liu Y, Liu J, et al. NEDD4L-mediated LTF protein degradation limits ferroptosis. Biochem Biophys Res Commun. 2020;531(4):581-7. https:// doi.org/10.1016/j.bbrc.2020.07.032.

46. Yang $\mathrm{ND}$, Tan $\mathrm{SH}, \mathrm{Ng} \mathrm{S}$, et al. Artesunate induces cell death in human cancer cells via enhancing lysosomal function and lysosomal degradation of ferritin. J Biol Chem. 2014;289(48):33425-41. https://doi.org/10.1074/jbc. M114.564567.

47. Eling N, Reuter L, Hazin J, et al. Identification of artesunate as a specific activator of ferroptosis in pancreatic cancer cells. Oncoscience. 2015;2(5):517-32. https://doi.org/10.18632/oncoscience.160 (Published 2015 May 2)

48. Hou $W$, Xie Y, Song $X$, et al. Autophagy promotes ferroptosis by degradation of ferritin. Autophagy. 2016;12(8):1425-8. https://doi.org/10.1080/ 15548627.2016.1187366.

49. Bai T, Wang S, Zhao Y, et al. Haloperidol, a sigma receptor 1 antagonist, promotes ferroptosis in hepatocellular carcinoma cells. Biochem Biophys Res Commun. 2017;491(4):919-25. https://doi.org/10.1016/j.bbrc.2017.07. 136.

50. Wu C, Zhao W, Yu J, et al. Induction of ferroptosis and mitochondrial dysfunction by oxidative stress in PC12 cells. Sci Rep. 2018;8(1):574. https:// doi.org/10.1038/s41598-017-18935-1 (Published 2018 Jan 12). 
51. Li C, Zhang Y, Liu J, et al. Mitochondrial DNA stress triggers autophagydependent ferroptotic death. Autophagy. 2021;17(4):948-60. https://doi. org/10.1080/15548627.2020.1739447.

52. Rong Y, Gao J, Kuang T, et al. DIAPH3 promotes pancreatic cancer progression by activating selenoprotein TrxR1-mediated antioxidant effects. J Cell Mol Med. 2021;25(4):2163-75. https://doi.org/10.1111/jcmm.16196.

53. Yuan LQ, Wang C, Lu DF, et al. Induction of apoptosis and ferroptosis by a tumor suppressing magnetic field through ROS-mediated DNA damage. Aging (Albany NY). 2020;12(4):3662-81. https://doi.org/10.18632/aging. 102836

54. Yang WS, Stockwell BR. Ferroptosis: death by lipid peroxidation. Trends Cell Biol. 2016;26(3):165-76. https://doi.org/10.1016/j.tcb.2015.10.014.

55. Conrad M, Kagan VE, Bayir H, et al. Regulation of lipid peroxidation and ferroptosis in diverse species. Genes Dev. 2018;32(9-10):602-19. https:// doi.org/10.1101/gad.314674.118.

56. Kuang F, Liu J, Xie Y, et al. MGST1 is a redox-sensitive repressor of ferroptosis in pancreatic cancer cells. Cell Chem Biol. 2021. https://doi.org/ 10.1016/j.chembiol.2021.01.006 (published online ahead of print, 2021 Jan 20)

57. Ye Z, Hu Q, Zhuo Q, et al. Abrogation of ARF6 promotes RSL3-induced ferroptosis and mitigates gemcitabine resistance in pancreatic cancer cells. Am J Cancer Res. 2020;10(4):1182-93 (Published 2020 Apr 1).

58. Hu N, Bai L, Dai E, et al. Pirin is a nuclear redox-sensitive modulator of autophagy-dependent ferroptosis. Biochem Biophys Res Commun. 2021;536:100-6. https://doi.org/10.1016/j.bbrc.2020.12.066.

59. Seibt TM, Proneth B, Conrad M. Role of GPX4 in ferroptosis and its pharmacological implication. Free Radic Biol Med. 2019;133:144-52. https:// doi.org/10.1016/j.freeradbiomed.2018.09.014.

60. Wei Y, Lv H, Shaikh AB, et al. Directly targeting glutathione peroxidase 4 may be more effective than disrupting glutathione on ferroptosis-based cancer therapy. Biochim Biophys Acta Gen Subj. 2020;1864(4): 129539 https://doi.org/10.1016/j.bbagen.2020.129539.

61. Wang H, Liu C, Zhao Y, et al. Inhibition of LONP1 protects against erastininduced ferroptosis in Pancreatic ductal adenocarcinoma PANC1 cells. Biochem Biophys Res Commun. 2020;522(4):1063-8. https://doi.org/10. 1016/j.bbrc.2019.11.187.

62. Ye Z, Zhuo Q, Hu Q, et al. FBW7-NRA41-SCD1 axis synchronously regulates apoptosis and ferroptosis in pancreatic cancer cells. Redox Biol. 2021;38: 101807. https://doi.org/10.1016/j.redox.2020.101807.

63. Badgley MA, Kremer DM, Maurer HC, et al. Cysteine depletion induces pancreatic tumor ferroptosis in mice. Science. 2020;368(6486):85-9. https://doi.org/10.1126/science.aaw9872.

64. Lelièvre P, Sancey L, Coll JL, et al. Iron dysregulation in human cancer: altered metabolism, biomarkers for diagnosis, prognosis, monitoring and rationale for therapy. Cancers (Basel). 2020;12(12):3524. https://doi.org/10. 3390/cancers12123524 (Published 2020 Nov 26).

65. El Hout M, Dos Santos L, Hamaï A, et al. A promising new approach to cancer therapy: targeting iron metabolism in cancer stem cells. Semin Cancer Biol. 2018;53:125-38. https://doi.org/10.1016/j.semcancer.2018.07. 009.

66. Moniaux N, Chakraborty S, Yalniz M, et al. Early diagnosis of pancreatic cancer: neutrophil gelatinase-associated lipocalin as a marker of pancreatic intraepithelial neoplasia. Br J Cancer. 2008;98(9):1540-7. https://doi. org/10.1038/sj.bjc.6604329.

67. Marcus DM, Zinberg N. Isolation of ferritin from human mammary and pancreatic carcinomas by means of antibody immunoadsorbents. Arch Biochem Biophys. 1974;162:493-501. https://doi.org/10.1016/00039861(74)90209-4.

68. Jeong SM, Hwang S, Seong $\mathrm{RH}$. Transferrin receptor regulates pancreatic cancer growth by modulating mitochondrial respiration and ROS generation. Biochem Biophys Res Commun. 2016;471(3):373-9. https://doi.org/ 10.1016/j.bbrc.2016.02.023.

69. Dai E, Han L, Liu J, et al. Ferroptotic damage promotes pancreatic tumorigenesis through a TMEM173/STING-dependent DNA sensor pathway. Nat Commun. 2020;11(1):6339. https://doi.org/10.1038/s41467-020-20154-8 (Published 2020 Dec 11)

70. Toshiyama R, Konno M, Eguchi H, et al. Association of iron metabolic enzyme hepcidin expression levels with the prognosis of patients with pancreatic cancer. Oncol Lett. 2018;15(5):8125-33. https://doi.org/10. 3892/ol.2018.8357.

71. Li C, Zhang Y, Cheng X, et al. PINK1 and PARK2 suppress pancreatic tumorigenesis through control of mitochondrial iron-mediated immunometabolism. Dev Cell. 2018;46(4):441-455.e8. https://doi.org/10.1016/j. devcel.2018.07.012.

72. Jiang P, Yang F, Zou C, et al. The construction and analysis of a ferroptosisrelated gene prognostic signature for pancreatic cancer. Aging (Albany NY). 2021;13(7):10396-414. https://doi.org/10.18632/aging.202801.

73. Liu J, Song X, Kuang F, et al. NUPR1 is a critical repressor of ferroptosis. Nat Commun. 2021;12(1):647. https://doi.org/10.1038/s41467-021-20904-2

(Published 2021 Jan 28).

74. Kuang F, Liu J, Li C, et al. Cathepsin B is a mediator of organellespecific initiation of ferroptosis. Biochem Biophys Res Commun. 2020;533(4):1464-9. https://doi.org/10.1016/j.bbrc.2020.10.035.

75. Liu C, Wang D, Zhan Y, et al. Switchable photoacoustic imaging of glutathione using $\mathrm{MnO} 2$ nanotubes for cancer diagnosis. ACS Appl Mater Interfaces. 2018;10(51):44231-9. https://doi.org/10.1021/acsami.8b14944.

76. Lv H, Zhen C, Liu J, et al. Unraveling the potential role of glutathione in multiple forms of cell death in cancer therapy. Oxid Med Cell Longev. 2019;2019:3150145. https://doi.org/10.1155/2019/3150145 (Published 2019 Jun 10).

77. Daher B, Parks SK, Durivault J, et al. Genetic ablation of the cystine transporter XCT in PDAC cells inhibits MTORC1, growth, survival, and tumor formation via nutrient and oxidative stresses. Cancer Res. 2019;79(15):3877-90. https://doi.org/10.1158/0008-5472.CAN-18-3855.

78. Liu Y, Wang Y, Liu J, et al. Interplay between MTOR and GPX4 signaling modulates autophagy-dependent ferroptotic cancer cell death. Cancer Gene Ther. 2021;28(1-2):55-63. https://doi.org/10.1038/ s41417-020-0182-y.

79. Song $X$, Liu J, Kuang F, et al. PDK4 dictates metabolic resistance to ferroptosis by suppressing pyruvate oxidation and fatty acid synthesis. Cell Rep. 2021;34(8):108767. https://doi.org/10.1016/j.celrep.2021.108767.

80. Tang R, Hua J, Xu J, et al. The role of ferroptosis regulators in the prognosis, immune activity and gemcitabine resistance of pancreatic cancer. Ann Transl Med. 2020;8(21):1347. https://doi.org/10.21037/atm-20-2554a.

81. Dai E, Han L, Liu J, et al. Autophagy-dependent ferroptosis drives tumorassociated macrophage polarization via release and uptake of oncogenic KRAS protein. Autophagy. 2020;16(11):2069-83. https://doi.org/10.1080/ 15548627.2020 .1714209$.

82. Zhang J, Sun H, Liu S, et al. Alteration of tumor-associated macrophage subtypes mediated by KRT6A in pancreatic ductal adenocarcinoma. Aging (Albany NY). 2020;12(22):23217-32. https://doi.org/10.18632/ aging.104091.

83. Brunner TB, Haustermans $K$, Huguet $F$, et al. ESTRO ACROP guidelines for target volume definition in pancreatic cancer. Radiother Oncol. 2021;154:60-9. https://doi.org/10.1016/j.radonc.2020.07.052.

84. Lei G, Zhang Y, Koppula P, et al. The role of ferroptosis in ionizing radiation-induced cell death and tumor suppression. Cell Res. 2020;30(2):14662. https://doi.org/10.1038/s41422-019-0263-3.

85. Cobler $\mathrm{L}$, Zhang $\mathrm{H}$, Suri $\mathrm{P}$, et al. $\mathrm{xCT}$ inhibition sensitizes tumors to $\gamma$-radiation via glutathione reduction. Oncotarget. 2018;9(64):32280-97. https://doi.org/10.18632/oncotarget.25794 (Published 2018 Aug 17).

86. Pan X, Lin Z, Jiang D, et al. Erastin decreases radioresistance of NSCLC cells partially by inducing GPX4-mediated ferroptosis. Oncol Lett. 2019;17(3):3001-8. https://doi.org/10.3892/ol.2019.9888.

87. Shibata Y, Yasui $H$, Higashikawa K, et al. Erastin, a ferroptosis-inducing agent, sensitized cancer cells to $X$-ray irradiation via glutathione starvation in vitro and in vivo. PLOS ONE. 2019;14(12):e0225931. https://doi.org/ 10.1371/journal.pone.0225931 (Published 2019 Dec 4).

\section{Publisher's Note}

Springer Nature remains neutral with regard to jurisdictional claims in published maps and institutional affiliations. 